\title{
Cell membrane rupture: A novel test reveals significant variations among different brands of tissue culture flasks
}

\author{
Ruy Tchao, Ph.D., \\ Department of Pharmaceutical Sciences, University of the Sciences, Philadelphia, PA 19104 \\ e-mail: r.tchao@usciences.edu
}

\begin{abstract}
Unanticipated errors in scientific research data can be attributed to the unwarranted assumption of uniformity in the polystyrene surface that is ubiquitously used in tissue culture flasks and dishes. We have shown that when adherent cells are subjected to fluid shear force, equivalent to rinsing the culture with a balanced salt solution, cells on some areas of the polystyrene surface will immediately rupture while still adherent on the surface. This heterogeneity on the polystyrene surface can cause unexpected variability in experimental results and in replicating experiments among labs. In this paper a novel quantitative method is described to measure the degree of heterogeneity on the polystyrene surface of tissue culture flasks. The results show significant variation among several brands of tissue culture flasks as well as large variability within the production lot of a manufacturer. The assay method involves loading the cells with a fluorescent marker that is released upon membrane rupture. Cell membrane rupture also causes the loss of marker proteins used in Westernblots. This novel assay method can be used to monitor the batch consistency and the manufacturing process of flasks and dishes. It may also be used to test new biomaterials.
\end{abstract}

Key words: polystyrene, tissue culture, cell membrane stability

\section{Introduction:}

The physiological functions of cells in tissues can be affected by physical and mechanical forces of the tissue and in the microenvironment. For example, osteocytes respond to mechanical stress of the bone and aortic endothelium responds to the hemodynamic forces from the blood circulation and may contribute to the location of atherosclerotic plaques (White and Frangos 2007) [1], and endothelium function (Johnson et al. 2011)[2] Therefore, understanding the interaction between cells, the matrix and external factors is essential for the development of new biomaterials. The behavior and functions of cells in-vitro are also affected by the microenvironment including the substratum. (reviewed by Schiller and Fässler 2013 [3], Wong 2004)[4]. For example, the substratum strain and rigidity affects cell motility (Plontnikov et al. 2012)[5], and fluid shear forces applied to endothelial cells causes upregulation of a number of signal transduction processes (Liu et al. 2014)[6].

Polystyrene (PS) is the material used ubiquitously in cell culture (Lerman et al. 2018) [7]. We have observed a phenomenon that certain areas of the PS surface on which cells adhere, cell membrane stability can be altered compared to the rest of the PS surface. When a fluid force such as the rinsing of cells after the removal of medium, is applied to the monolayer, on certain areas of the flask or dishes, the plasma membrane of adherent cells will rupture immediately, while the cells are still adherent to the PS surface (Tchao 1996) [8]. This effect is not observed with cells grown on glass or collagen coated surfaces, but is observable with fibronectin or laminin coated surfaces. It appears to be related to the stress in the PS polymer that developed during the manufacturing process and can be demonstrated with birefringence pattern in the plastic (Lau and Tchao 2007)[9]. This fluid shear effect was first observed 
with a squamous cells line, NBT-II. It seems to occur with several epithelial cell lines, including normal keratinocytes, human mammary tumor cell lines (MCF-10A), human prostate tumor cell lines (DU-145) but it is not observed with fibroblastic cells or epithelial cells that have tight junctions, such as the MDCK cell line and some epithelial cells that show more mesenchymal morphology such as MDA MB231 (unpublished data).

For molecular and biochemical analysis of the cultured cells, after the removal of the culture medium, cells are generally rinsed with a buffered salt solution such as PBS or HBSS. We have shown that the fluid force over the cell monolayer during the rinsing procedure can cause some cells to rupture, therefore a concern is that the rinsing process can cause the loss of house-keeping proteins such as GAPDH or $\beta$-actin or $\alpha$-tubulin that are used as standards in Westernblots. Specific target molecules in the cytoplasm may be lost, leading to variability in repeating experimental results, as well as the irreproducibility of the results from another lab that uses different flasks/dishes. In addition, in comparative studies using two different cell lines, errors may occur owing to the differential responses to fluid shear of the two cell lines, for example MCF10A, a near normal breast cell line that responds to fluid shear and MDA-MB231, representing a triple negative breast cancer cell line that does not respond to fluid shear.

Variability in PS surface has been demonstrated in PS flasks and dishes by physical measurements (Zeiger et al. 2013) [10]. The usual test for PS surface suitability as well as for other biomaterials, is its ability to support cell growth and spreading. Most manufactured PS surfaces can satisfy this requirement. However, subtle heterogeneity of the PS surface causing adherent cells to respond to fluid shear has been observed in our lab and this paper describes a novel biologically based method to quantitatively determine the percent of the PS surface in tissue culture flasks and dishes that contribute to cell membrane rupture due to fluid shear force. This novel method can be helpful for the end user to compare the consistency of batches of plastic ware. This method can also be used by PS manufacturers to monitor the quality of their production, including any changes in the manufacturing process, and for testing new biomaterials.

Methods and Materials:

Samples of T-25 flasks were obtained from selected manufacturers (Chemglass, Corning, Falcon, Greiner, Nunc, Santacruz, Sarstedt, TPP,).

NBT-II cells are routine culture in DMEM/F12 medium supplemented with $10 \%$ fetal bovine serum, all obtained from ThermoFisher.

HanksBalanced salt solution (HBSS) with $\mathrm{Ca}++$ and $\mathrm{Mg}++$ without phenol red, is obtained from ThermoFisher (cat number 14025092).

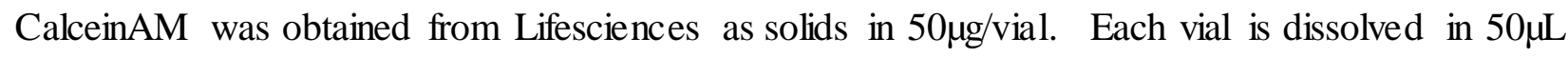
DMSO to give a stock solution of $1 \mathrm{mM}$ concentration. For incubation with cells, the stock solution is diluted to $0.5-4 \mu \mathrm{M}$ in HBSS. Preliminary experiments using $0.5 \mu \mathrm{M}$ CalceinAm have shown that DMSO from $0.1-0.4 \%$ do not affect CalceinAM uptake by cells. 
CyQuant assay kit was purchased from Lifesciences , InVitrogen ® CyQUANT Cell Proliferation Assay Kit.

Anti GAPDH labelled with Alexa488 was purchased from ThermoScientific (cat \#MA5-15738-D488) .

Zeiss Axioscope equipped with Polaroid color imaging system is used to record fluorescent images.

NIH Image J program is used to merge fluorescent images.

Fluorescence is quantitatively measured in a plate reader, Cyto2300 at excitation $485 \mathrm{~nm}$ and emission $590 \mathrm{~nm}$.

Preliminary experiments to determine CalceinAM uptake and the effect of cell number were performed in Corning 24-well plates.

\section{Fluid shear Procedure applied to T-25 flasks:}

Based on the preliminary results on CalceinAM loading, the following procedure has been adopted to measure the percentage of shear in T-25 flasks by various manufacturers.

Culture medium is aspirated from the T-25 flasks and $2 \mathrm{ml}$ cold HBSS is added to rinse the cells and then aspirated. Previous experiments have shown that cold HBSS does not produce the fluid shear effect of membrane rupture in cells $[8,9]$.

$2 \mathrm{ml}$ CalceinAM, $2 \mu \mathrm{M}$, is added to each T-25 flask and incubated at $37^{\circ} \mathrm{C}$ for 60 minutes.

At the end of CalceinAM incubation, $2 \mathrm{ml}$ Cold HBSS is added to each well and extracellular CalceinAM is removed by aspiration, and the flasks are rinsed twice with $2 \mathrm{ml}$ cold HBSS each time.

The second rinse of $2 \mathrm{ml} \mathrm{HBSS}$ is kept for fluorescence measurement, that serves as the background for the specific flask.

Then, warm HBSS $2 \mathrm{ml}$ is added and the flask is incubated at $37^{\circ} \mathrm{C}$ for 10 minutes to equilibrate the warm HBSS solution and the flask. Fluid shear is applied to the cells by rocking the flask back and forth, and the fluid is collected for fluorescence measurement, representing the fluorescence of sheared cells. After the removal of the "fluid shear" solution, $2 \mathrm{ml}$ of $0.1 \%$ Triton X100 in HBSS neutralized to $\mathrm{pH} 7.2$, is added and incubated at room temp for 10 minutes to release the total fluorescent Calcein in the remaining cells. The Triton solution is collected for fluorescence measurement. All fluorescence measurements were done in a fresh 24 well plate.

The following formula is used to calculate the percent of shear in each flask :

Sheared cell washings + Triton X100 released fluorescence $=$ Total fluorescence

Fluid Sheared cell solution fluorescence / Total fluorescence $=\%$ heterogeneity on PS surface

For each run, 3-4 flasks of each brand are used. The experiments are repeated $3 \times(n=3)$ for statistical analysis of significance. 


\section{Results:}

Initially it was thought that the release of housekeeping proteins such as GAPDH used in WesternBlots could be used to quantify the fluid shear effect. However, qualitative fluorescence imaging of Alexa488 labelled antiGAPDH showed that after fluid shear, in some cells although some protein in the cytoplasm may be lost, but GAPDH actually may be condensed or be retained in the nucleus of the cells.

In other cells the loss of cytoplasmic GAPDH was partial as shown in Fig 1.

\section{Fig 1 GAPDH staining of fluid shear cells}

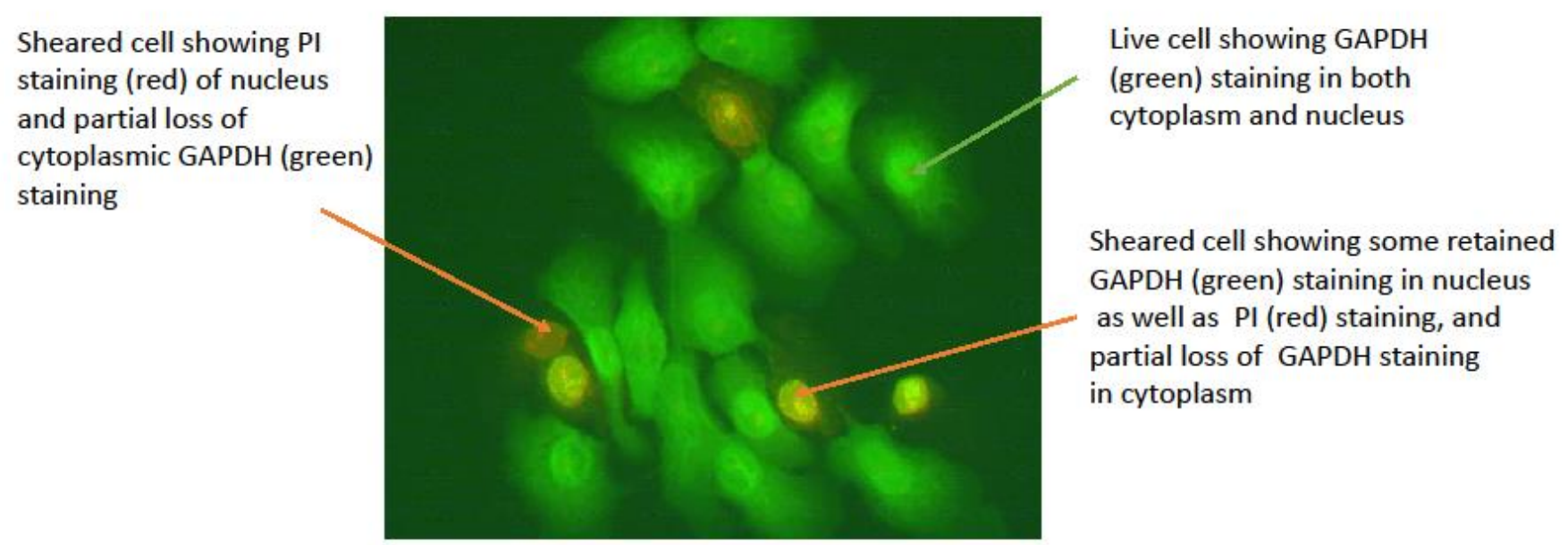

Fig 1 Fluid sheared effect: live cells are stained with anti-GAPDH antibody (green fluorescence) Membrane ruptured cells are stained with PI (red fluorescence) However, GAPDH is retained in the nuclei of some ruptured cells, yielding orange/yellow in merged image, there is partial loss of cytoplasmic GAPDH in ruptured cells

Fig 1 shows that upon fluid shear, in some cells, cytoplasmic GAPDH was lost and some was condensed in and around the nucleus. The sheared cells were stained with Propidium Iodide immediately after fluid shear and then fixed for 5 minutes in 10\% formalin for antibody (anti-GAPDH) staining. Interestingly, the short fixation with $10 \%$ formalin did not affect the cells already stained with PI. Since not all cells lose the marker proteins GAPDH equally throughout the culture, GAPDH cannot be used to quantify the effect of fluid shear. Therefore, a smaller molecule that is not bound inside the cells will be a better choice to quantify the fluid shear effect. CalceinAM, a non-fluorescent molecule is easily taken by live cells, and inside the cell, CalceinAM is converted by non-specific esterases into a fluorescent molecule (Haugland 2002) [11]. 
Preliminary experiments show qualitatively that CalceinAM loaded cells show green fluorescence, and upon fluid shear, the ruptured cells lose the Calcein green fluorescence, and the nucleus can be stained with propidium iodide. In Figure 2a, panel A shows Calcein labelled cells before shear and panel B show application of fluid shear causing a large patch of ruptured cells that can be stained with Propidium Iodide as show in panel 3. The staining of live cells with Calcein and dead cells with propidium iodide is the basis of the Live/Dead Assay®developed by Invitrogen.

The juxtaposition of live and ruptured cells shown in figure $2 \mathrm{~b}$ suggests that the heterogeneity of polystyrene is at the micro or nano level. This present a challenge to the detection of polystyrene surface irregularity by physical methods. Methods such as AFM can detect surface irregularities but the correlation to cellular effect of fluid shear is a challenge (unpublished data).

Figure 2a Fluid shear on CalceinAm loaded NBT-II cells

A

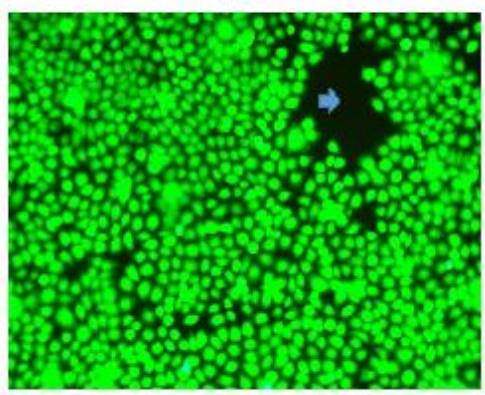

CalceinAM loaded cells before shear The dark area without cells is indicated by an arrow (compare with sheared cells in panel B)

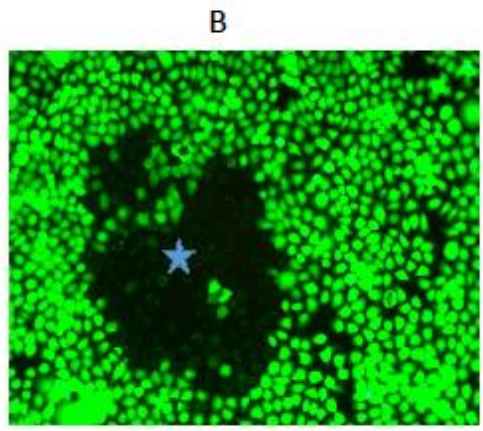

Culture after fluid shear, showing a patch of cells indicated by a star that have lost Calcein fluorescence, appearing as "ghost cells".

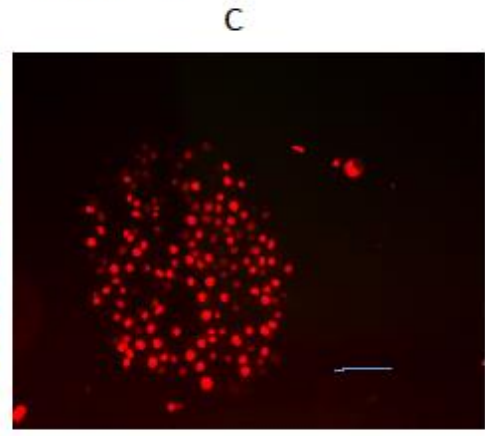

Propidium lodide $(\mathrm{PI})$ staining of membrane ruptured cells in the same area indicated by a star in panel B. Bar $=0.1 \mathrm{~mm}$

Fig 2a The effect of fluid shear in CalceinAM loaded cells.

Panel A. NBT-Il cells are grown in T-25 flask to near confluence and are incubated in $2.0 \mu \mathrm{M}$ CalceinAM for 60 minutes. CalceinAM is converted to green fluorescent Calcein.

Panel B. Fluid shear is then applied, those cells that rupture have lost the fluorescence, the area is indicated by a star. Panel C. The nuclei of cell membrane ruptured cells can be also stained with $5 \mu \mathrm{g} / \mathrm{ml}$ Propidium lodide, (red fluprescence). The magnification in all three panels is the same, represented by the bar $(=0.1 \mathrm{~mm})$ in panel $\mathrm{C}$. 


\section{Figure $2 \mathrm{~b} \quad$ Fluid shear effect on NBT-II cells Calcein labeled live cells and PI labeled dead cells}

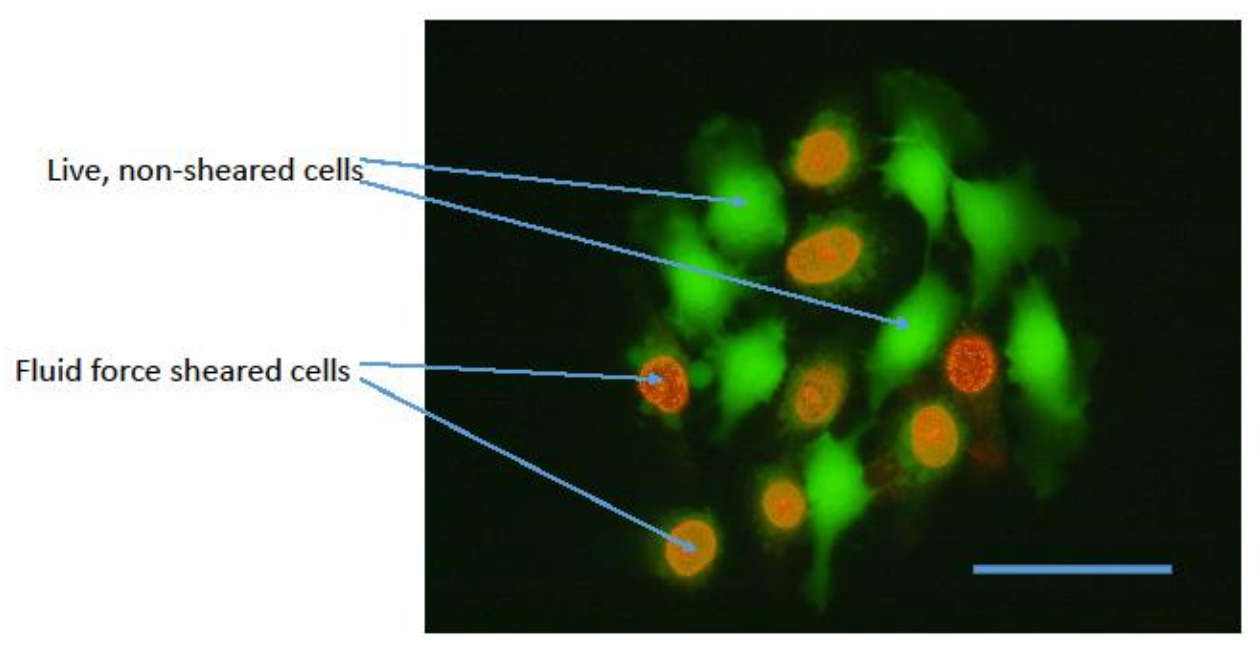

Cells are grown on Polystyrene plastic

bar $=50 \mu \mathrm{M}$

Fig $2 \mathrm{~b} \quad$ A merged image at higher magnification of sheared cells, showing the loss of Calcein fluorescence and the nuclei staining of Propidium iodide, as well as some intact cells with Calcein fluorescence. Note the juxtaposition of the live and dead cells. Also visible are the membrane "blebs" indicating the loss of membrane integrity in sheared cells.

To test the efficiency of extracting total Calcein fluorescence in cells, cells are grown in a 24-well plate to $70-80 \%$ confluent cultures. Cells are then loaded with CalceinAM $(2-5 \mu \mathrm{M})$ in $1 \mathrm{ml}$ HBSS. The Calcein fluorescence is measured in a plate reader. After the removal of the CalceinAm incubation solution, the wells are rinsed with cold HBSS. The total Calcein fluorescence in the cells is extracted completely with Triton X100 as represented by percent of calcein fluorescence measured in the 24-well plate and the results are shown in Table 1.

Table 1: Triton extraction of preloaded CalceinAM fluorescence.

\begin{tabular}{|l|l|l|}
\hline & Triton extraction \% & SD \\
\hline Expt 1 & 98.8 & 0.04 \\
\hline Expt 2 & 102.7 & 0.09 \\
\hline Expt 3 & 95.0 & 0.13 \\
\hline
\end{tabular}

Table 1 Triton extracted fluorescence was compared to the fluorescence in monolayer cells in 24 well plates, after 60 minutes incubation with $2 \mu \mathrm{M}$ CalceinAM. Each experiment consists of 12 wells of cells. 
After rinsing the cells with cold HBSS, $1 \mathrm{ml} 0.1 \%$ neutralized triton x100 was added. After incubation at room temp for 10 minutes, the triton solutions were removed into fresh wells for fluorescence measurements.

Experiments were done to show that the uptake of CalceinAM is both time and cell number dependent, as well as dependency on CalceinAM concentrations.

Using 24-well plates, Fig. 3a shows that CalceinAM uptake is time dependent at various cell densities.

Fig. $3 \mathrm{~b}$ shows that when the uptake is normalized to a certain cell number, the uptake with time shows a linear regression of uptake with time at various cell densities.

This means that the uptake results are comparable over a range of seeding cell density, so that repeated experiments can be compared to each other.

Fig. 4 shows the uptake is dependent on CalceinAM concentrations. For uptake experiment to compare T-25 flasks manufactured by different companies, the concentration of $2 \mu \mathrm{M}$ CalceinAM is chosen.

\section{Figure 3a Calcein AM Uptake and Cell Density}

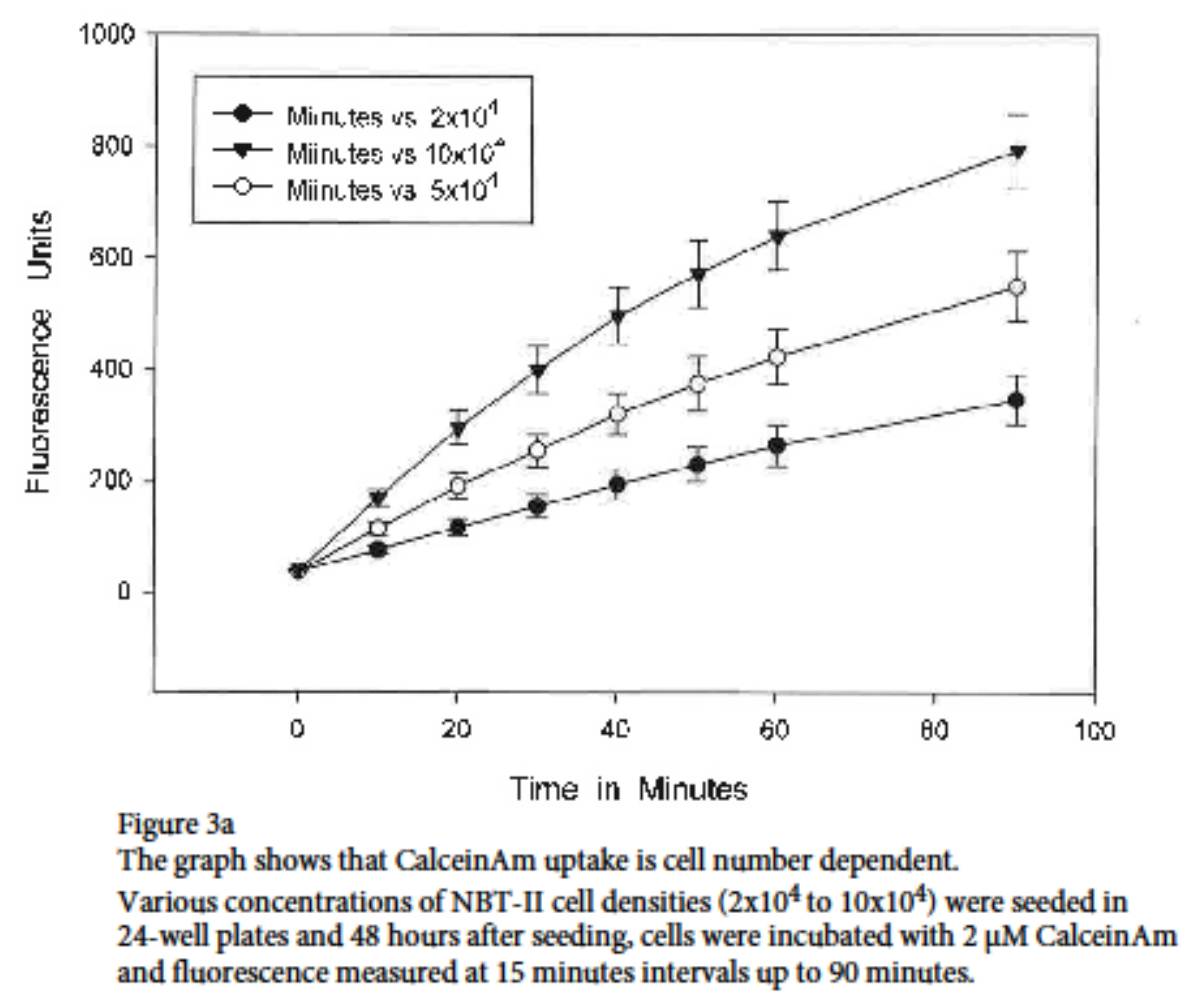


Fig 3b Uptake of $2 \mu \mathrm{M}$ CalceinAM by NBT-Il cells

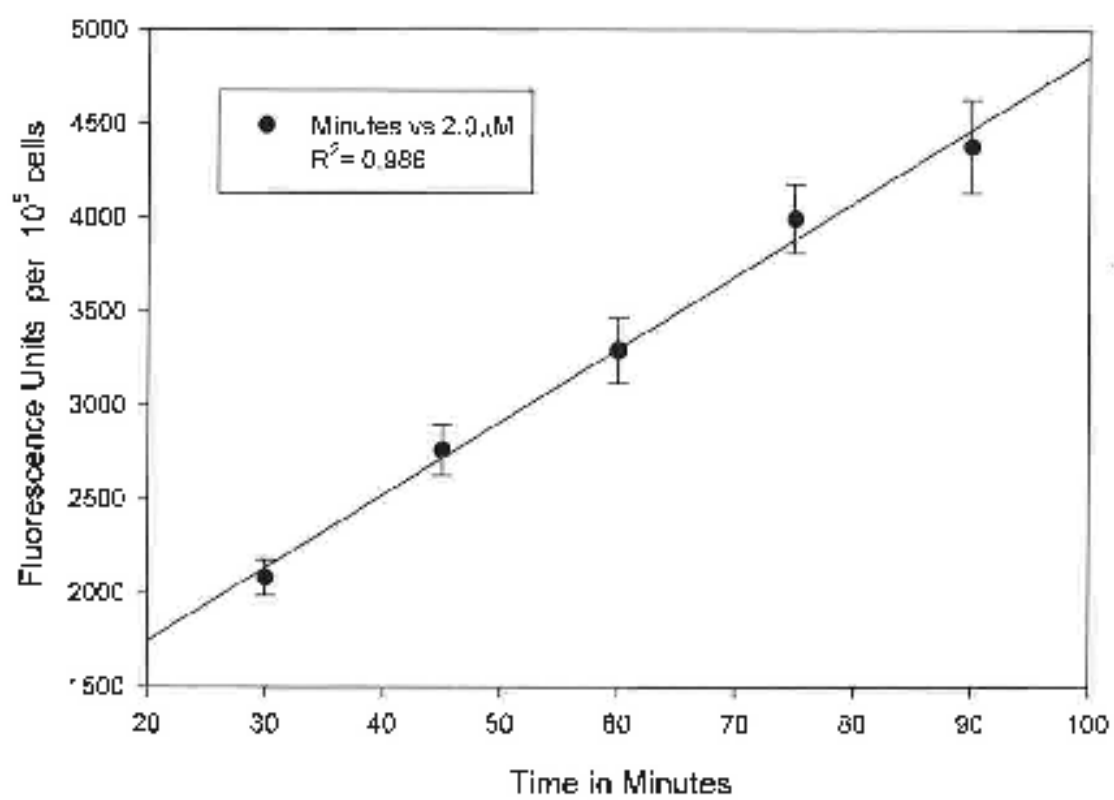

Figure $3 \mathrm{~b}$ This figure shows the regression plot of $2 \mu \mathrm{M}$ of Calcein Am uptake by NBT-II cells seeded at various cell concentrations $\left(2 \times 10^{4}-10 \times 10^{4}\right.$ per well).

The number of cells in each well was determined by CyQuant assay.

The regression curve shows linear uptake from 30-90 minutes when the fluorescence is

normalized to $10^{5}$ cells. Subsequent experiments on the shear effect on NBT-II cells in T-25 flasks were performed after 60 minutes uptake of $2 \mu \mathrm{M}$ CalceinAM. 
Figure 4 Wptake of CalceinAM by NBT-II cells

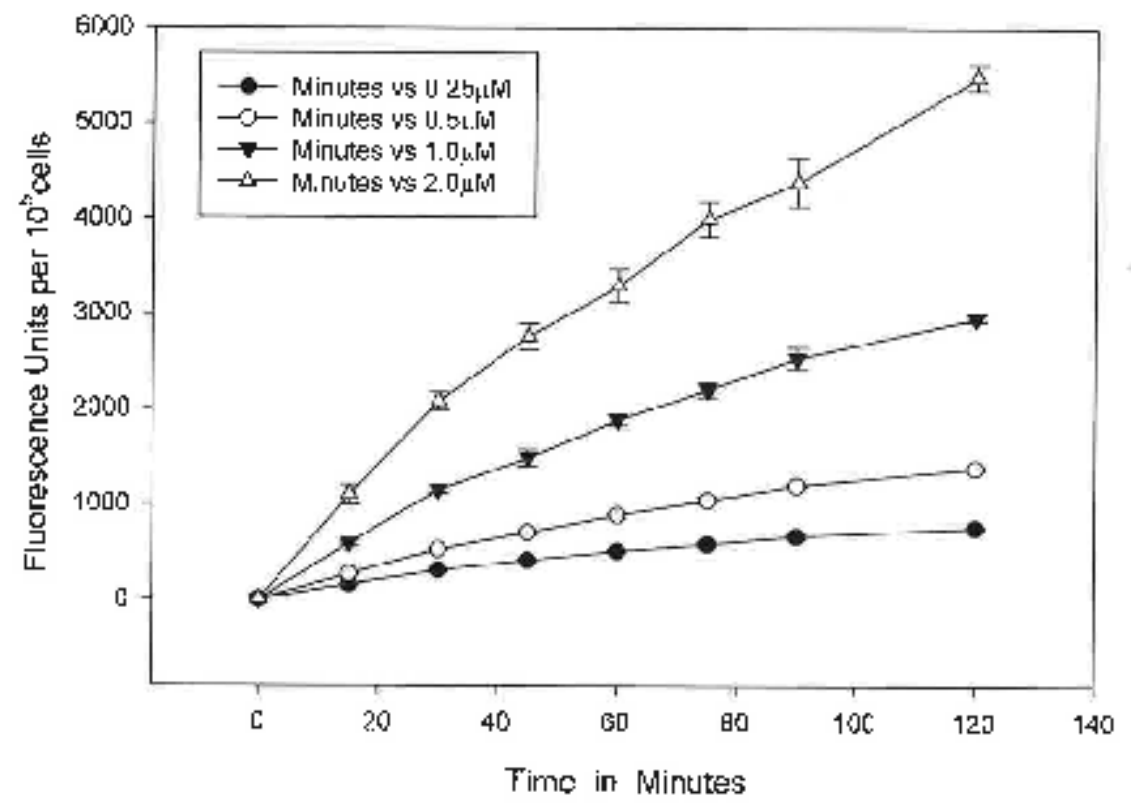

Figure 4 Shows NBT-II cells uptake of CalceinAm at various concentrations of CalceinAm.

Cells were seeded at $5 \times 10^{4}$ cells per well and the uptake experiments were done 48 hours after seeding. The arbitrary florescence units measured in each experiment were normalized using

CyQuant assay of cell number in each well.

The error bars represent the SEM from 3 separate experiments each involve 5 replicate wells

of CalceinAm uptake measurements, and the average value was used for statistical calculations. 
A comparison of T-25 flasks from various manufacturers is shown in Table 2a. The results show that there is considerable difference among the manufacturers. In addition, flask aging may be a factor in the cellular response to fluid shear. However, specialized treatment to alter the PS surface charges (CellBind ${ }^{\circledR}$ ) did not prevent the fluid shear effect. In comparison to NBT-II cells, MDCK cells do not exhibit fluid shear effect as shown in Table $2 b$.

Table 2a Fluid shear effect of NBT-Il cells grown on T-25 of various manufacturers

\begin{tabular}{|l|l|l|l|}
\hline Manufacturer & Cat \# & Lot \# & \% Fluid Shear Effect +/- sem \\
\hline Chemglass(CellTreat) & 229331 & $110723-218$ & $* 10.5+/-0.5$ \\
\hline Corning (regular) & 3056 & 09815045 & $* 14.1+/-0.6$ \\
\hline Corning (Cell-Bind) & 3289 & 22005005 & $8.7+/-0.5$ \\
\hline Falcon & 353108 & 5249004 & $* 13.6+/-1.4$ \\
\hline Greiner & 690170 & 08050140 & $* 11.3+/-0.2$ \\
\hline Nunc & 136196 & 136985 & $* 20.6+/-0.3$ \\
\hline SantaCruz & SC-200262 & $110423-218$ & $* 11.8+/-0.5$ \\
\hline Sarstedt & 831810 & 6065081 & $* 13.4+/-1.1$ \\
\hline TPP & 90025 & 20150141 & $4.5+/-1.3$ \\
\hline
\end{tabular}

The results shown in table 2 a represent 3 separate runs on the flasks therefore the SEM represent $n=3$. For each run, 3-4 flasks of each brand are used.

* indicates that fluid shear effect in flask is significantly greater $(\mathrm{p}<0.05)$ than that exhibited by MDCK cells as shown in table $2 b$.

Table $2 b$ Fluid shear effect of MDCK cells grown on T-25 flasks ( 5 flasks each):

\begin{tabular}{|l|l|}
\hline Manufacturer & $\%$ shear $+/-$ sd \\
\hline Corning & $2.4+/-0.4$ \\
\hline Greiner & $3.3+/-0.4$ \\
\hline
\end{tabular}

\section{Discussion :}

It is a long and generally held view by researchers that for tissue culture studies, particularly stem cell cultures, one should not change the manufacturer of tissue culture flasks and dishes lest it might affect the reproducibility of the results. This means that during manufacturing of tissue culture flasks, some factor or factors can introduce heterogeneity onto the surface, causing difference among the manufacturers during the injection molding of the plastic flasks or dishes. 
This paper describes a novel biological assay based on the fluid shear effect on adherent cells that we described in earlier studies, to determine the heterogeneity of the PS surface used in tissue culture. As cells rupture, cytoplasmic content will be released. Therefore the potential loss of molecules of interest, such as signal transduction proteins may vary when different PS dishes are used, and can lead to irreproducibility among experiments. Indeed, this paper shows that the amount of the fluid shear rupture of cell membrane is variable among various manufacturers of the culture flasks, representing a potential variable when the investigator switches to a different manufacturer for PS flasks and dishes.

In life sciences, reproducibility of research data is essential, particularly in pre-clinical research. A recent paper analyzed the cumulative cost of irreproducible preclinical research to be $\$ 28$ billion in the US alone (Freedman et al. 2015) [12]. The paper further showed that of the four categories of preclinical irreproducibility, biological reagents and reference material accounted for $36.1 \%$ of the total cost. Tissue culture technique is used widely in preclinical research. However, standardization in the PS used in flasks and dished does not exist. It is provocative to suggest that because of this fluid shear effect of cells grown on PS, variability in the results of analyzing small molecules may occur in different laboratories. Battiston et al. (2011) [13] have shown that tissue culture polystyrene from different manufacturers differ considerably in protein binding and the release of cytokines by monocytes interacting with the polystyrene. They used several physical methods, including contact angle measurement, scanning electron microscopy, and X-ray photoelectron spectroscopy to analyze the PS surface of tissue culture dishes manufactured by Sarstedt, Wisent and Becton Dickinson. They conclude that some particular surface chemical properties of the PS can influence the experimental outcome of monocyte interaction on the surface.

The mechanism of cell response to the physical and chemicals nature of the substratum is complex (reviewed by Ohashi K., S. Fujiwara and K. Mizuno 2017) [14]. Several cell functions such as motility and differentiation are influenced by the cell sensing the mechanical properties of the substratum such as rigidity and smoothness (review by Vogel and Sheetz 2006) [15]. The exact mechanism involved in cell membrane rupture upon fluid shear on certain areas of the PS surface is not known. However, it is expected that cell adhesion proteins such as cadherins, integrins and focal adhesion kinases may be involved (reviewed by Schiller and Fässler 2013 [3]. In addition, cytoskeleton proteins such as actin may also be involved (Lau, unpublished data). We have observed that MDCK and LLCpk1 cells that exhibit tight junctions do not rupture under fluid shear. However, when MDCK cells are treated with EGTA to disrupt the tight junction, cells then respond to fluid shear effect (unpublished observations). This suggests that membrane composition in addition to rho signaling in cytoskeleton structure may also play a role.

The results shown in Table 2a included the Corning CellBind flasks (Ryan2008) [16] that exhibited reduced fluid shear effect but did not eliminate the effect suggesting that PS surface charge may not be the only factor causing the heterogeneity in the PS surface. The TPP brand showed much less effect of fluid shear effect, however, the variations (SEM) among the lot tested were very large.

Fig. $3 b$ illustrated that the dead and live cells are juxtaposed with each other, suggesting that the sensing of PS surface by cells may be at micro-meter or nano-meter level. Previous studies have shown that the edges of the flasks and dishes, and in areas related to the injection port, there is strong birefringence under cross-polarized light suggesting that PS molecular orientation may contribute to the 
sensitivity of cells to fluid shear. It has been shown that during the injection mold process, as the PS polymer solidifies, shrinkage can affect the dimension of the final product but also has a large effect on the residual stress distribution of the product (Jansen et al. 1998) [17], and can be predicted (Titomanlio and Jansen 1996) [18]. Indeed, application of stress to a disc cast from a polystyrene solution showed birefringence and cells grown on such surface demonstrated the fluid shear effect (Lau and Tchao 2007) [9]. We speculate that the molecular orientation of the surface of the PS polymer is similar to that of the bulk PS that shows birefringence. Therefore, the fluid shear effect of cells may be due to cell interaction with the molecular orientation of the PS polymer at the surface. The liquid crystal alignment of a polymer surface can be modified by rubbing (Stohr and Samant 1999) [19], and the resulting molecular orientation of the PS surface has been studied (Briggman et al 2001) [20]. The assay described in this paper can be easily used to study the modification of PS surfaces.

The biocompatibility or biological responses of biomaterial needs to be evaluated with various parameters (Anderson 2013)[21]. The most common aspect of biological evaluation of biomaterials is cytotoxicity, with occasional reference to effect on cell growth and cell adhesion. The cell line most used is a fibroblast. Our observation of the fluid shear on cell membrane represent a unique aspect of interaction between the substratum and adherent epithelial cells that may yield different aspects of biomaterial/cell interaction in the development of biomaterial.

The integrity of cell membrane under fluid shear may have relevance in vivo. In vascular physiology, mechano-transduction is important in maintaining normal endothelium (EC) function (Hahn and Schwartz 2009) [22]. Under laminal flow, the EC produces anti-inflammatory molecules whereas under disturbed flow (turbulent flow) such as at arterial bifurcation and branch points, the EC can produce pro-inflammatory molecules leading to a propensity for atherogenesis (Heo et al. 2015) [23]. Therefore, any pathological changes in the basement membrane such as the accumulation of lipids, under disturbed flow, the endothelial cell could exhibit membrane damage including cell rupture and cell death, that could enhance the pro-inflammatory reaction. In cancer metastasis, invasive cancer cells enter the blood circulation in order to spread to distant sites. Circulating cancers cells have been shown not to survive the hemodynamic shear force (Weiss et al. 1985) [24]. Therefore only a small percentage of initially viable cancer cells progress to form metastasis (Weiss 1989) [25], leading to the observation that the metastatic process is inefficient (Weiss 1990) [26]. However, an alternative view on the effect of fluid shear effect on metastatic cells is that the tumor cells that are adherent to the endothelium may be protected from the fluid shear force. Therefore, studying the tumor cell membrane and understanding its adhesion to surfaces can be useful if the sensitivity of tumor cells to fluid shear force can be increased to enhance tumor cell death.

\section{Conclusions:}

The polystyrene surface used in tissue culture flasks and dishes have been known to be variable as shown by various physical measurements (Zeiger et al. 2013) [10]. The heterogeneity of the polystyrene surface in flasks and dishes may present a variable that contributes to the variability in repeated experiments as well as irreproducibility of results among various labs. However, there is at present no easy method for a research investigator to determine the consistency of the flasks from different manufacturers. This paper describes a simple biologically based method using a fluorescent dye to label cultured cells and the rupture of cell membrane in response to a fluid shear force similar to 
rinsing the cell culture. The researcher can then check the consistency of the culture flask and dishes from different manufacturers. The method is also useful for the manufacturers to verify the consistency of their products and to determine any change in the polystyrene surface resulting from modifications in the molding process.

Acknowledgement: Research is supported by funds from the Department of Pharmaceutical Sciences, University of the Sciences in Philadelphia, USA

Declaration of interest: None

The raw data required to reproduce these findings are available to download from:https://drive.google.com/drive/u/0/folders/13uj_LdCt4JQD0HTs06S6P2959Dw_pOn5 


\section{References:}

1. White C.R., J.A. Frangos, The shear stress of it all: the cell membrane and mechnochemical transduction. Phil. Trans. R. Soc. B 362 (2007) 1459-67.

2. Johnson B.D., K.J. Mather, J.P. Wallace, Mechanotransduction of shear in endothelium: basic studies and clinical implications. Vascular Medicine 16(5), (2011) 365-77.

3. Schiller N.B., R. Fässler Mechanosensitivity and compositional dynamics of cell-matrix adhesions. EMBO reports 14(6), (2013) 509-19.

4. Wong, J.Y., J.B. Leach, X.Q. Brown, Balance of chemistry, topography, and mechanics at the cell-biomaterial interface: Issues and challenges for Assessing the role of substrate mechanics of cell response. Surface Science 570 (1), (2004) 119-133.

5. Plotnikov S., A. Pasapera, B. Sabass, C. Waterman. Force fluctuations within focal adhesions mediate ECM-rigidity sensing to guide directed cell migration. Cell 151, (2012) 1513-27

6. Liu B., S.Lu, Y-L. Hu, X. Liao, M. Ouyang, Y. Wang. RhoA and membrane fluidity mediates the spatially polarized Src/FAK activation in response to shear stress. Scientific Reports 4, (2014) 7008-14, DOI, 10.1038/srep07008

7. Lerman M.J, J. Lembong, S. Muramoto, G. Gillen, J.P. Fisher. The evolution of polystyrene as a cell culture material. Tissue Engineering: Part B, 24 (5), (2018) 359-372.

doi:10.1089/ten.teb.2018.0056

8. Tchao, R. Fluid shear force and turbulence-induced cell death in plastic tissue culture flasks. In-vitro Toxicology 9(1), (1996) 93-100.

9. Lau, J.Y., R. Tchao, Stressed polystyrene causes increased membrane sensitivity of adherent cells to fluid shear force: Technical note. Europ. Cells and Materials 14 (2007) 40-44.

10. Zeiger, A.S., B. Hinton, K.J. Van Vliet, Why the dish makes a difference: Quantitative comparison of polystyrene culture surfaces. Acta Biomaterialia 9 (2013) 7354-7361

11. Haugland, R.P. Handbook of fluorescent probes and research products. Molecular Probes, Eugene, OR, 2002.

12. Freedman,L.P., I.M. Cockburn, T.S. Simcoe, The economics of reproducibility in preclinical research. $\quad$ PLOS Biol. 13 (6) (2015) e1002165 doi: 10.1371/journal.pbio.1002165

13. Battiston K.G., J.E.McBane, R.S. Labow, J.P. Santerre 2012. Differences in protein binding and cytoskeleton release from monocytes on commercially sources tissue culture polystyrene. Acta Biomaterialia 8 (2012) 89-98

14. Ohashi, K., S. Fujiwara, K. Mizuno, Roles of the cytoskeleton, cell adhesion and rho signaling in mechanicaosensing and mechnotransduction. J. Biochem. 161(3) (2017) 245-254

15. Vogel, V., M. Sheetz. Local force and geometry sensing regulate cell functions. Nature Reviews, Molecular Cell Biology, 7 (2006) 265-275. 
16. Ryan, J.A. Evolution of cell culture surfaces. BioFiles 3(8) (2008) 21-27

17. Jansen K.M.B., R. Pantani, G. Titomanlio As-Molded shrinkage measurements on polystyrene injection molded products. Polymer Eng. and Science, 38 (2) (1998) 254-64

18. Titomanlio, G., K.M.B. Jansen, In-mold shrinkage and stress prediction in injection molding. Polymer Eng. and Sci. 36 (15) (1996) 2041-2049.

19. Stöhr, J., M.G. Samant, Liquid crystal alignment by rubbed polymer surface: a microscopic bond orientation model. J. Electr. Spectr. and related Phenom. 98-99 (1999) 189-207

20. Briggman, K.A., J.C. Stephenson, W.E. Wallace, L.J. Richter. Absolute molecular orientation distribution of the polystyrene surface. J. Phys. Chem. B, 105 (2001) 2785-91

21. Anderson J.M, Future challenges in the in-vitro and in-vivo evaluation of biomaterial biocompatibility. Regenrative Biomaterials 73-77 (2016) 73-76 doi: 10.1093/rb/rbw001

22. Hahn C., M.A. Schwartz, 2009. Mechanotransduction in vascular physiology and atherogenesis. Nat Rev Mol Cell Biol. 10 (1) (2009) 53-62

23. Heo, K-S, K. Fujiwara, J-i. Abe, Shear Stress and atherosclerosis. Mol. Cells 37 (2014) 435-440.

24. Weiss L., D.S. Dimitrov, M. Angelova, 1985. The hemodynamic destruction of intravascular cancer cells in relation to myocardial metastasis. Proc. Natl. Acad. Sci. USA 82 (1985) 5737-5741

25. Weiss L., FW. Orr, K.V. Honn Interactions between cancer cells and the microvasculature: a rate-regulator for metastasis. Clinical \& Experimental Metastasis, 7 (1989) 127-167

26. Weiss L., Metastatic inefficiency. Adv. Cancer Res. 54 (1990) 159-211. 


\section{Summary Statement of significance:}

This paper describes a novel test protocol based on fluid shear rupture of adherent cells in certain areas of tissue culture flasks and dishes. The fluid shear force, similar to rinsing the cultures with a salt solution HBSS, causes the cell membrane to rupture on certain areas of the polystyrene and lose the cytoplasmic content, including marker proteins used in Westernblots. The significance of the paper is to provide a simple cell based test to compare quantitatively the products from various manufacturers. The scientific impact and interest to scientists in the field of biomaterial is to recognize that errors due to variable amount of cell rupture may exist in the normal process of rinsing the cell culture for biochemical analysis. 